\section{Evaluación del valor pronóstico de la relación neutrófilos/linfocitos en cáncer de mama de subtipos agresivos}

\author{
XIMENA MIMICA ${ }^{1}$, FRANCISCO ACEVEDO ${ }^{2}$, DAVID ODDO ${ }^{3}$, \\ CAROLINA IBÁÑEZ ${ }^{2}$, LIDIA MEDINA ${ }^{2}$, ALEXIS KALERGIS $^{4}$, \\ MAURICIO CAMUS' ${ }^{1}$, CÉSAR SÁNCHEZ ${ }^{2}$
}

\section{Neutrophil/lymphocyte ratio in complete blood count as a mortality predictor in breast cancer}

Background: The white blood cell count is one of the most sensitive markers associated with inflammation. The neutrophil/lymphocyte count ratio may be an independent factor for breast cancer mortality. Aim: To assess the predictive value of the neutrophil/lymphocyte ratio for mortality in breast cancer. Material and Methods: Review of the database of a cancer center of a University hospital. Patients with infiltrating breast cancer treated between 1997 and 2012 were selected. The pathology type and lymph node involvement were obtained from the pathology report. The expression of estrogen, progesterone and Human Epidermal Growth Factor Receptor 2 (HER2) was determined by immunohistochemistry or in situ fluorescent hybridization (FISH). The absolute peripheral neutrophil and lymphocyte counts were obtained from a complete blood count obtained at least three months before treatment. Patients were followed for a median of 61 months (range 1-171). Results: From 323 eligible patients, after excluding those in stage IV and those without an available complete blood count, 131 patients were analyzed ( 81 with negative receptors and 117 HER2 enriched). The neutrophil/lymphocyte ratio was similar in both types of tumors (2.1 and 1.91 respectively). Twenty two patients died during follow-up. Surviving patients with HER2 enriched tumors had a lower neutrophil/lymphocyte ratio than those who died (1.79 and 3.21 respectively, $p<0.01$ ). In a multivariate analysis, including age, tumor stage and lymph node involvement as confounding factors, the neutrophil/lymphocyte ratio was still significantly associated with a risk of death with a hazard ratio of 2.56. Conclusions: A high neutrophil/lymphocyte ratio in the complete blood count can be a predictor of death in breast cancer.

(Rev Med Chile 2016; 144: 691-696)

Key words: Breast Neoplasms; Inflammation; Prognosis.

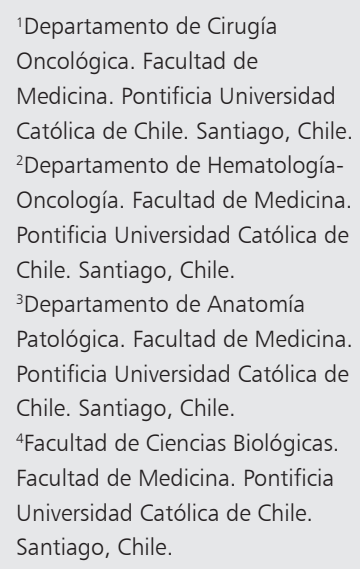

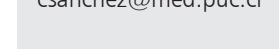

$\mathrm{E}$ 1 cáncer de mama (CM) es la neoplasia más común en mujeres ${ }^{1}$ y la principal causa de muerte por cáncer en chilenas ${ }^{2}$. Mientras la mayoría de las neoplasias de mama son estrógeno-dependientes, hasta $25 \%$ no expresan el receptor de estrógenos (RE) y/o progesterona
$(\mathrm{RP})^{3}$. Estas últimas pueden sobreexpresar HER2 o no expresar ninguno de estos tres receptores (RE, RP y HER2), habitualmente estudiados en muestras de CM. Tumores que no expresan receptores hormonales (RE- y RP-) y sin expresión de HER2 se denominan TN. Por otra parte, aquellos 
con sobreexpresión exclusiva de HER2 se llaman HER2 enriquecidos ${ }^{4}$. Estos dos subtipos tumorales han demostrado ser clínicamente más agresivos, cursan con recurrencias precoces y con una mayor frecuencia de enfermedad visceral ${ }^{4}$.

Durante el proceso de carcinogénesis, la inflamación originada por el tumor o su microambiente puede favorecer el crecimiento tumoral y su progresión metastásica ${ }^{5}$. Marcadores inflamatorios elevados, como la proteína C reactiva (PCR) e interleukina-6 (IL6), se han relacionado a una reducción de la supervivencia en pacientes con $\mathrm{CM}^{6,7}$. Los glóbulos blancos (GB) periféricos son uno de los biomarcadores inflamatorios más útiles en la práctica clínica y pueden ser cuantificados fácilmente a través de un hemograma. Aunque los glóbulos blancos pueden estar en rango normal, la relación neutrófilos/linfocitos (RAN/RAL) se ha utilizado como parámetro para determinar inflamación sistémica ${ }^{7,8}$. La relación RAN/RAL parece ser un predictor independiente de la mortalidad por CM y progresivamente ha sido reconocida como un indicador de mal pronóstico en varios tipos de cáncer ${ }^{9,10}$. Sin embargo, la consistencia y la magnitud del impacto pronóstico de la relación RAN/RAL no ha sido estudiado en diferentes subtipos de $\mathrm{CM}^{10}$. El objetivo de este estudio es evaluar el valor pronóstico de la razón RAN/RAL en subtipos agresivos de CM.

\section{Material y Métodos}

\section{Población}

Estudio retrospectivo realizado en el Centro del Cáncer de la Pontificia Universidad Católica de Chile, en Santiago, Chile. El estudio fue aprobado por el Comité de Ética de la Pontificia Universidad Católica de Chile. Se analizó nuestra base de datos de pacientes con CM infiltrante tratadas entre los años 1997 y 2012, excluimos pacientes con etapa IV (metastásicos) al momento del diagnóstico.

\section{Revisión patológica}

La información del subtipo histológico y el compromiso linfonodal fueron obtenidos del informe patológico. El estado de expresión de RE, RP y HER2 fue determinado a través de inmunohistoquímica (IHQ). El valor de corte para determinar la negatividad de RE y RP fue de $<1 \%$ de células tumorales con tinción nuclear. Con- sideramos como HER2 positivos todos aquellos tumores con puntuación de 3 cruces $(+++)$ en IHQ. En caso de ser considerados 2 cruces $(++)$, se realizó un estudio de hibridación fluorescente in situ (FISH) para HER2, procedimiento realizado en la mayoría de los casos (este estudio no era obligatorio en nuestro centro hasta el año 2006). El estadio al momento del diagnóstico se determinó de acuerdo con el sistema TNM del American Joint Committee on Cancer Staging Manual 2010 (séptima edición $)^{11}$.

\section{Subtipos clínico-patológicos}

Los tumores se clasificaron en 2 subtipos de acuerdo a marcadores clásicos: HER-2 enriquecidos (RE-, RP- y HER2 sobreexpresado) y TN (RE-, RP- y HER2 negativo), como ya hemos publicado previamente ${ }^{12}$.

\section{Relación neutrófilos/linfocitos}

La relación RAN/RAL se definió como el recuento absoluto de neutrófilos dividido por el recuento absoluto de linfocitos, medidos en $\mathrm{mm}^{3}$. Este dato fue obtenido del hemograma más cercano al diagnóstico de $\mathrm{CM}$, dentro de los tres meses previo al inicio de la terapia local (cirugía, radioterapia) o sistémica.

\section{Análisis estadístico}

Se utilizó $\chi^{2}$ (o prueba exacta de Fisher) para evaluar variables categóricas. Usamos prueba de Wilcoxon para comparar medianas. La sobrevida global (SG) fue calculada de acuerdo al método de Kaplan-Meier y comparadas mediante prueba de Log-Rank. Se utilizó ANOVA para realizar análisis multivariado. Consideramos una diferencia estadísticamente significativa cuando el valor de $\mathrm{p}$ fue menor o igual a 0,05 . Todos los datos fueron analizados utilizando la versión de $\mathrm{IBM}^{\circledR}{ }^{\circledR}$ SPSS $^{\circledR} 21$.

\section{Resultados}

En nuestra base de datos identificamos 323 tumores definidos como de subtipo agresivo: 206 TN y 117 HER2 enriquecidos. Luego de excluir pacientes con etapa IV y aquellos sin información disponible del recuento de neutrófilos y linfocitos dentro de los 3 meses previos al inicio del tratamiento, analizamos 130 pacientes; 81 con tumores TN y 49 con tumores HER 2 enriquecidos. Las ca- 
Tabla 1. Características clínico-patológicas de 130 pacientes con tumores de mama de subtipos agresivos

\begin{tabular}{|c|c|c|c|}
\hline Características & TN & HER2 enriquecidos & $\mathbf{p}$ \\
\hline Edad, años & 55 (29-84) & 56 (39-78) & 0,4 \\
\hline $\begin{array}{l}\text { Estadio según TNM } \\
\text { II } \\
\text { III }\end{array}$ & $\begin{array}{ll} & (81) \\
34,5 \% & (28) \\
39,5 \% & (32) \\
26 \% & (21)\end{array}$ & $\begin{array}{l}(49) \\
(16) \\
(16) \\
(17)\end{array}$ & 0,54 \\
\hline $\begin{array}{l}\text { Compromiso nodal } \\
\text { Sí } \\
\text { No }\end{array}$ & $\begin{array}{ll} & (81) \\
35 \% & (28) \\
65 \% & (53)\end{array}$ & $\begin{array}{l}(48) \\
(27) \\
(21)\end{array}$ & 0,016 \\
\hline $\begin{array}{l}\text { Quimioterapia } \\
\text { Sí }\end{array}$ & $\begin{array}{ll} & (75) \\
86 \% & (65)\end{array}$ & $\begin{array}{l}(39) \\
(28)\end{array}$ & 0,052 \\
\hline
\end{tabular}

TN: Triple negativos. HER2: Receptor del factor de crecimiento epidérmico tipo 2.

racterísticas de las pacientes se muestran en la Tabla 1. La mediana de edad fue 55 años para mujeres con tumores TN y de 56 años para mujeres con tumores HER2 enriquecidos. La distribución por etapas fue similar en ambos grupos, con casi $2 / 3$ de las pacientes en etapas I y II. Un mayor número de pacientes con tumores HER2 enriquecidos tenían compromiso linfonodal (56\% vs 35\%, para HER positivo y $\mathrm{TN}$, respectivamente, $\mathrm{p}=0,016$ ). Mientras más pacientes con tumores TN recibieron quimioterapia ( $86 \%$ vs $57 \%$ ), esta diferencia no fue estadísticamente significativa $(\mathrm{p}=0,052)$. Con una mediana de 61 meses de seguimiento (1172) no se ha alcanzado la mediana de sobrevida. La sobrevida global estimada a 5 años fue de $85 \%$ para tumores TN y de $83 \%$ para tumores HER2 positivos $(\mathrm{p}=0,2)$ (Figura 1$)$.

Con respecto a la evaluación de los glóbulos blancos en el hemograma, pacientes con tumores TN tuvieron recuentos más elevados de neutrófilos que pacientes con tumores HER2 enriquecidos (RAN 4000 vs 3400, $\mathrm{p}=0,013$ ), sin diferencias en el RAL (Tabla 2).

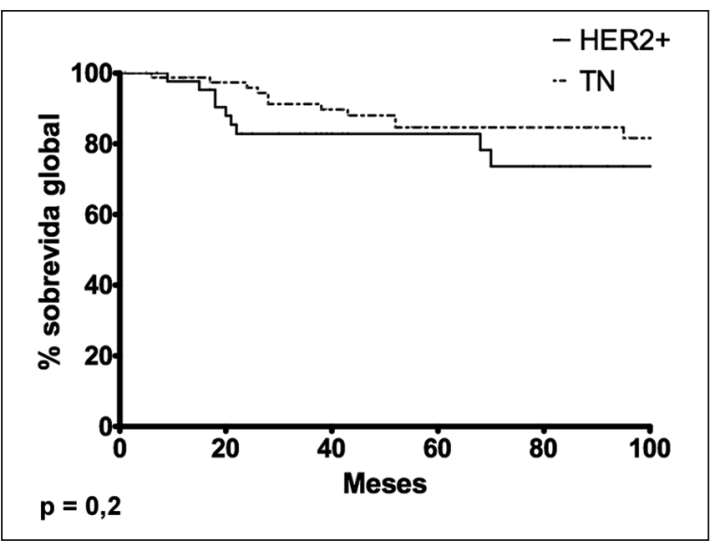

Figura 1. Sobrevida global de pacientes con cáncer de mama triple negativo (TN) y HER2 enriquecidos (HER2 +).

La mediana de la relación RAN/RAL fue similar en ambos grupos 2,1 vs 1,91 para tumores TN y HER2 enriquecidos, respectivamente, $\mathrm{p}=0,2$. Tumores TN y HER2 enriquecidos en etapa III tenían mayor relación RAN/RAL comparados con tumores del mismo subtipo en etapas I y II

Tabla 2. Número de glóbulos blancos en el hemograma de 130 pacientes con CM de subtipos agresivos

\begin{tabular}{|lccccc|}
\hline Glóbulos blancos $\mathbf{~ m m}^{\mathbf{3}}$ & \multicolumn{2}{c}{ TN } & \multicolumn{2}{c}{ HER2 enriquecidos } & p \\
\hline Leucocitos & \multicolumn{4}{c}{ Mediana (rango) } & 0,02 \\
Neutrófilos & 6.700 & $(3.200-14.900)$ & 5.900 & $(1.200-10.400)$ & 0,013 \\
Linfocitos & 4.000 & $(900-12.500)$ & 3.400 & $(612-6.800)$ & 0,6 \\
\hline
\end{tabular}

TN: Triple negativos. HER2: Receptor del factor de crecimiento epidérmico tipo 2. 
Tabla 3. Relación RAN/RAL en pacientes con CM de subtipos agresivos, según etapa, compromiso ganglionar y sobrevida

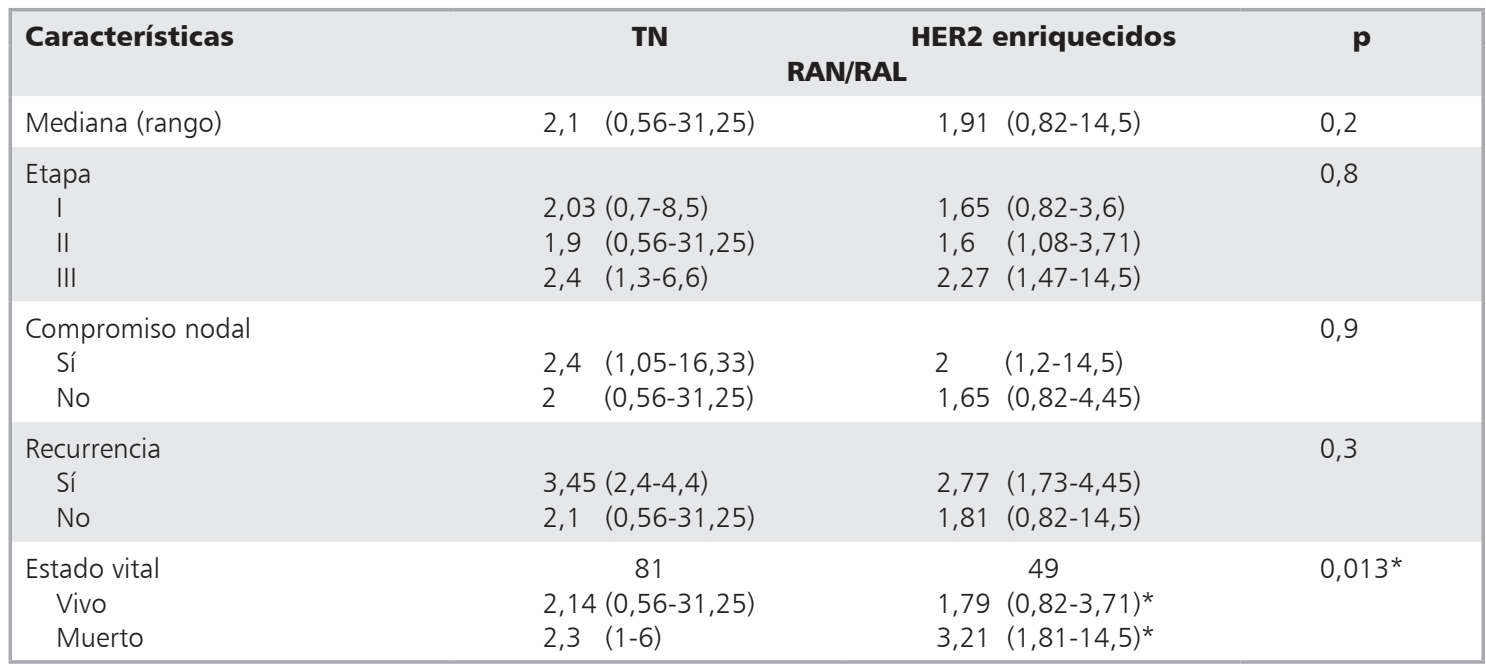

* Diferencia estadísticamente significativa para tumores HER2 positivo. TN: Triple negativos. HER2: Receptor del factor de crecimiento epidérmico tipo 2. RAN/RAL: Recuento absolutos de neutrófilos/Recuento absoluto de linfocitos.

(Tabla 3); sin embargo, esta diferencia no fue estadísticamente significativa. Tampoco observamos diferencia entre el compromiso nodal y RAN/RAL. Pacientes con recurrencia de la enfermedad tenían RAN/RAL más elevado, sin embargo, esta diferencia no logró significancia estadística (Tabla 2).

Veintidós pacientes fallecieron durante el seguimiento, 14 del grupo de tumores TN y 8 en grupo de HER2 enriquecidos. Dentro de este grupo de pacientes, aquellos con tumores HER2 enriquecidos

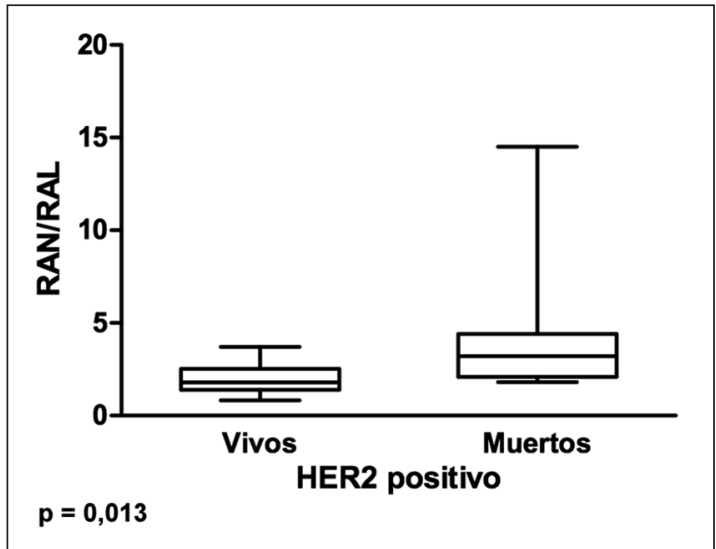

Figura 2. Estado vital de pacientes con cáncer de mama HER2 enriquecidos, según relación recuento absoluto de neutrófilos/ recuento absoluto de linfocitos. que han fallecido presentaban una relación RAN/ RAL más elevada que aquellos que permanecían vivos, RAN/RAL 1,79 en vivos comparado con 3,21 para fallecidos, $\mathrm{p}=0,00034$ (Figura 2). En el análisis multivariado considerando edad, etapa, compromiso linfonodal y relación RAN/RAL, sólo la relación RAN/RAL estuvo significativamente asociada a muerte (HR: 2,56; $\mathrm{p}=0,013$ ).

$\mathrm{Al}$ evaluar estos datos según el tratamiento recibido, se observó que en el subgrupo de pacientes no tratados con trastuzumab (un anticuerpo monoclonal contra el receptor de HER2), el valor RAN/RAL persiste mayor en pacientes fallecidos $(p=0,0023)$. En el subgrupo tratado con trastuzumab sólo hubo 2 pacientes fallecidos, lo que limita el análisis de estos datos (Tabla 4).

Tabla 4. Relación RAN/RAL en pacientes HER2 enriquecido con uso o no de trastuzumab y su estado vital

\begin{tabular}{|lccc|}
\hline & $\begin{array}{c}\text { Vivos } \\
\text { (N/RAN-RAL*) }\end{array}$ & $\begin{array}{c}\text { Muertos } \\
\text { (N/RAN-RAL*) }\end{array}$ & Total \\
Trastuzumab & $15(1,82)$ & $2(2,47)$ & 17 \\
No trastuzumab & $25(1,83)$ & $6(2,65)$ & 31 \\
Total & 40 & 8 & 48 \\
\hline
\end{tabular}

*Medianas. N: Número de pacientes. RAN/RAL: Recuento absolutos de neutrófilos/Recuento absoluto de linfocitos. 


\section{Discusión}

La inflamación es parte relevante de la carcinogénesis, dado que contribuye al estrés oxidativo que causa daño celular conducente a alteraciones en el genoma, resultando en alteraciones de la expresión génica como en la acumulación de mutaciones ${ }^{5,9}$.

Parámetros medibles habitualmente en sangre y que reflejan la respuesta inflamatoria sistémica, tales como PCR, hipoalbuminemia, citoquinas, niveles de leucocitos y sus subtipos, han sido asociados a pronóstico en varias neoplasias. Adicionalmente, marcadores bioquímicos de respuesta inflamatoria se han incorporado en las puntuaciones de pronóstico para varios tipos de cáncer ${ }^{8,13,14}$. La inflamación inducida por los cambios genéticos de la célula neoplásica o producida por el estroma tumoral puede ser parte importante de la evolución de lesiones pre-neoplásicas y neoplásicas ${ }^{15}$. Mientras la exposición a estradiol y factores hormonales es relevante en la carcinogénesis de CM $\mathrm{RE}+$, los fenómenos inflamatorios están presentes en todo el espectro de subtipos de $\mathrm{CM}^{6,16,17}$. Noh y cols. ${ }^{8}$ describieron en un estudio retrospectivo el valor de la relación RAN/RAL en CM. Analizaron 442 pacientes con RAN/RAL elevado y mostraron una menor sobrevida libre de enfermedad específica en este grupo de pacientes, principalmente en tumores definidos como Luminales A.

Azab y cols. evaluaron el valor pronóstico de RAN /RAL y de la relación recuento de plaquetas/ linfocitos, en la sobrevida de pacientes con CM. En 437 pacientes analizados, aquellos con el percentil más elevado de RAN/RAL tuvieron mayor mortalidad. Interesantemente la relación RAN/ RAL fue un factor pronóstico superior al recuento de plaquetas $^{18}$.

Recientemente, Chen y cols. realizaron un metaanálisis para establecer el valor pronóstico de la relación RAN/RAL en CM. Analizaron 8 estudios que incluían 4.293 pacientes. Sus resultados también confirman el valor de RAN/RAL como un factor pronóstico en $\mathrm{CM}^{19}$. Templenton y cols. ${ }^{14}$ analizaron 100 estudios de tumores sólidos, que incluían 40.559 pacientes; una relación RAN/ RAL > 4 se asoció a peor sobrevida en todos los subgrupos, sitios y etapas de la enfermedad.

Los linfocitos tienen un rol clave en la inmuno-vigilancia y en la inducción de la respuesta inmune adquirida, la cual puede restringir la proliferación celular neoplásica y el proceso de metástasis. La presencia de linfocitos infiltrantes de tumores ha sido descrita en variados tipos de neoplasias y relacionada a pronóstico ${ }^{20}$. Además, la normalización de la linfopenia tras la quimioterapia de altas dosis se asocia a un mejor pronóstico en pacientes con $\mathrm{CM}^{21}$.

Se ha observado que un aumento en los niveles de neutrófilos podría inhibir la actividad citolítica de linfocitos $\mathrm{T}$ y de las células natural killer, así como suprimir la proliferación de los linfocitos $\mathrm{T}^{22}$.

La relación RAN/RAL, por tanto, podría ser un parámetro asociado al desbalance de la actividad antitumoral en el micro-ambiente tumoral.

Nuestro trabajo apoya que una relación RAN/ RAL alta en pacientes con tumores HER 2 enriquecidos se asocia a una mayor mortalidad. Otras experiencias recientes proponen este parámetro como factor de riesgo de recidiva local. (Williams GJ, et al. Neutrophil lymphocyte ratio and breast cáncer: A future prognostic indicator for outcome after surgery (2015, dic). (Poster presentado en San Antonio Breast Cancer Symposium. San Antonio. Texas. Estados Unidos de Norteamérica).

Debemos indicar, que nuestro estudio tiene varias limitaciones, como son su naturaleza retrospectiva, un tamaño muestral escaso, tratamientos no uniformes, lo que limita sus conclusiones y dificulta el análisis de subgrupos. Además, analizamos sólo un hemograma, sin evaluar otros parámetros inflamatorios.

En conclusión, nuestros resultados están en concordancia con la literatura ${ }^{14}$ donde se sugiere un valor pronóstico a la relación RAN/RAL en tumores HER2 enriquecidos, en los que la inmunogenicidad es relevante.

\section{Referencias}

1. De Santis C, Ma J, Bryan L, Jemal A. Breast cancer statistics, 2013. CA Cancer J Clin 2014; 64 (1): 52-62.

2. Itriago L, Silva N, Cortés G. Cáncer en Chile y el mundo: una mirada epidemiológica, presente y futuro. Rev Med Clin Condes 2013; 24 (4): 531-52.

3. Goldhirsch A, Winer EP, Coates AS, Gelber RD, Piccart-Gebhart M, Thürlimann B, et al. Personalizing the treatment of women with early breast cancer: highlights of the St Gallen International Expert Consensus on the Primary Therapy of Early Breast Cancer 2013. Ann Oncol 2013; 24 (9): 2206-23. 
Valor pronóstico de la relación RAN/RAL en cáncer de mama - X. Mimica et al

4. Metzger-Filho O, Sun Z, Viale G, Price K N, Crivellari D, Snyder R D, et al. Patterns of Recurrence and outcome according to breast cancer subtypes in lymph node-negative disease: results from international breast cancer study group trials VIII and IX. J Clin Oncol 2013; 31 (25): 3083-90.

5. Okada F. Inflammation-related carcinogenesis: current findings in epidemiological trends, causes and mechanisms. Yonago Acta Med 2014; 57 (2): 65-72.

6. Baumgarten SC, Frasor J. Minireview: Inflammation: an instigator of more aggressive estrogen receptor (ER) positive breast cancers. Mol Endocrinol 2012; 26 (3): 360-71.

7. Roxburgh CSD, McMillan DC. Cancer and systemic inflammation: treat the tumour and treat the host. Br J Cancer. Nature Publishing Group; 2014; 110 (6): 140912.

8. Noh H, Eomm M, Han A. Usefulness of pretreatment neutrophil to lymphocyte ratio in predicting disease-specific survival in breast cancer patients. J Breast Cancer 2013; 16 (1): 55-9.

9. Elinav E, Nowarski R, Thaiss CA, Hu B, Jin C, Flavell RA. Inflammation-induced cancer: crosstalk between tumours, immune cells and microorganisms. Nat Rev Cancer 2013; 13 (11): 759-71.

10. Urrejola GI, Bambs CE, Espinoza MA, Gellona J, Zúñiga AM, Molina ME, et al. [An elevated neutrophil/lymphocyte ratio is associated with poor prognosis in stage II resected colon cancer]. Rev Med Chile 2013; 141 (5): 602-8.

11. Edge SB, Compton CC. The American Joint Committee on Cancer: the 7th Edition of the AJCC Cancer Staging Manual and the Future of TNM. Ann Surg Oncol 2010; 17 (6): 1471-4.

12. Sánchez C, Acevedo F, Petric M, Galindo H, Domínguez F, León A, et al. Cáncer de mama metastásico. Caracterización de una cohorte según subtipos. Rev Med Chile 2014; 142 (4): 428-35.

13. Taniguchi K, Karin M. IL-6 and related cytokines as the critical lynchpins between inflammation and cancer.
Semin Immunol 2014; 26 (1): 54-74.

14. Templeton AJ, McNamara MG, Šeruga B, Vera-Badillo FE, Aneja P, Ocaña A, et al. Prognostic role of neutrophil-to-lymphocyte ratio in solid tumors: A systematic review and meta-analysis. J. Natl. Cancer Inst 2014. p. dju124-dju124.

15. Colotta F, Allavena P, Sica A, Garlanda C, Mantovani A. Cancer-related inflammation, the seventh hallmark of cancer: Links to genetic instability. Carcinogenesis 2009; 30 (7): 1073-81.

16. Simpson ER, Brown KA. Obesity and breast cancer: Role of inflammation and aromatase. J Mol Endocrinol 2013; 51 (3): T51-9.

17. Iyengar NM, Hudis CA, Dannenberg AJ. Obesity and inflammation: new insights into breast cancer development and progression. Am Soc Clin Oncol Educ Book 2013; 33: 46-51.

18. Azab B, Shah N, Radbel J, Tan P, Bhatt V, Vonfrolio $\mathrm{S}$, et al. Pretreatment neutrophil/lymphocyte ratio is superior to platelet/lymphocyte ratio as a predictor of long-term mortality in breast cancer patients. Med Oncol 2013; 30 (1): 432.

19. Chen J, Deng Q, Pan Y, He B, Ying H, Sun H, et al. Prognostic value of neutrophil-to-lymphocyte ratio in breast cancer. FEBS Open Bio 2015; 5: 502-7.

20. Salgado R, Denkert C, Demaria S, Sirtaine N, Klauschen F, Pruneri G, et al. The evaluation of tumor-infiltrating lymphocytes (TILs) in breast cancer: recommendations by an International TILs Working Group 2014. Ann Oncol 2014; 26 (2): 259-71.

21. Nieto Y, Shpall EJ, McNiece IK, Nawaz S, Beaudet J, Rosinski S, et al. Prognostic analysis of early lymphocyte recovery in patients with advanced breast cancer receiving high-dose chemotherapy with an autologous hematopoietic progenitor cell transplant. Clin Cancer Res 2004; 10 (15): 5076-86.

22. Pillay J, Kamp VM, van Hoffen E, Visser T, Tak T, Lammers J-W, et al. A subset of neutrophils in human systemic inflammation inhibits $\mathrm{T}$ cell responses through Mac-1. J Clin Invest 2012; 122 (1): 327-36. 